\title{
THE TOTAL ACQUISITION NUMBER OF THE RANDOMLY WEIGHTED PATH
}

\author{
Anant GodBole \\ Department of Mathematics and Statistics \\ East Tennessee State University \\ e-mail: godbolea@mail.etsu.edu \\ ElizABETH KeLley \\ Department of Mathematics \\ University of Minnesota \\ e-mail: ekelley@g.hmc.edu \\ EMILY KURTZ \\ Department of Mathematics \\ Wellesley College \\ e-mail: ekurtz@wellesley.edu \\ PaWee Prąat \\ Department of Mathematics \\ Ryerson University \\ e-mail: pralat@ryerson.ca
}

AND

YIGUANG ZHANG

Department of Applied Mathematics and Statistics

The Johns Hopkins University

e-mail: yzhan132@jhu.edu

\begin{abstract}
There exists a significant body of work on determining the acquisition number $a_{t}(G)$ of various graphs when the vertices of those graphs are each initially assigned a unit weight. We determine properties of the acquisition
\end{abstract}


number of the path, star, complete, complete bipartite, cycle, and wheel graphs for variations on this initial weighting scheme, with the majority of our work focusing on the expected acquisition number of randomly weighted graphs. In particular, we bound the expected acquisition number $E\left(a_{t}\left(P_{n}\right)\right)$ of the $n$-path when $n$ distinguishable "units" of integral weight, or chips, are randomly distributed across its vertices between $0.242 n$ and $0.375 n$. With computer support, we improve it by showing that $E\left(a_{t}\left(P_{n}\right)\right)$ lies between $0.29523 n$ and $0.29576 n$. We then use subadditivity to show that the limiting ratio $\lim E\left(a_{t}\left(P_{n}\right)\right) / n$ exists, and simulations reveal more exactly what the limiting value equals. The Hoeffding-Azuma inequality is used to prove that the acquisition number is tightly concentrated around its expected value. Additionally, in a different context, we offer a non-optimal acquisition protocol algorithm for the randomly weighted path and exactly compute the expected size of the resultant residual set.

Keywords: total acquisition number, Poissonization, dePoissonization, Maxwell-Boltzman and Bose-Einstein allocation.

2010 Mathematics Subject Classification: 05C75.

\section{REFERENCES}

[1] D. Bal, P. Bennett, A. Dudek and P. Prałat, The total acquisition number of random graphs (2016), arXiv:1402.2854.

[2] E. Czabarka, M. Marsili and L.A. Székely, Threshold functions for distinct parts: revisiting Erdős-Lehner, in: Information Theory, Combinatorics, and Search Theory (in Memory of Rudolph Ahlswede), H. Aydinian, F. Cicalese and C. Deppe (Eds.), Springer-Verlag, Lecture Notes in Comput. Sci. 7777 (2013) 463-471. doi:10.1007/978-3-642-36899-8_22

[3] D.E. Lampert and P.J. Slater, The acquisition number of a graph, Congr. Numer. 109 (1995) 203-210.

[4] T. LeSaulnier, N. Prince, P. Wenger, D. West and P. Worah, Total acquisition in graphs, SIAM J. Discrete Math. 27 (2013) 1800-1819. doi: $10.1137 / 110856186$

[5] P. Wenger, Fractional acquisition in graphs, Discrete Appl. Math. 178 (2014) 142-148. doi:10.1016/j.dam.2014.06.010

[6] D. West, N. Prince and P. Wenger, Unit acquisition in graphs, preprint.

[7] P. Prałat, $\mathrm{C}++$ program and results can be found at http://www.math.ryerson.ca/ pralat/. 